\title{
An Analysis of the Impact of the Change in Scoring System on Home Field Advantage Soccer Leagues
}

\author{
Tuğbay Inan \\ School of Sport Science and Technology, Dokuz Eylül University, Izmir, Turkey
}

Copyright $\bigcirc 2018$ by authors, all rights reserved. Authors agree that this article remains permanently open access under the terms of the Creative Commons Attribution License 4.0 International License

\begin{abstract}
The impact of the scoring systems on home field advantage in the highest-level Turkish soccer division, Turkish Super League, between the 1959-1960 and 2016-2017 seasons, was aimed to be examined in this study. 2-point system was used in Turkish Soccer Leagues between 1959 and 1987. Since 1987-1988 season, the 3 -point system has been started to be used in Turkey. A total of 33,244 games were analyzed and it was revealed that there was a remarkable decline in home field advantage rates after the change in scoring system in 1987-1988 season. In the Turkish Soccer League, the average home field advantage rate was $66,39 \%$ before the change in the scoring system, while this rate was found to be $61,40 \%$ after the change in scoring system $(t=5,041$, $p=0,000)$. The results indicate that there is significant difference between 2-point and 3-point systems in terms of total points of home team at home $(\mathrm{t}=-11,83, \mathrm{p}=0,000)$. The results of this study were also compared with a relevant study of the highest-level soccer league in Spain.
\end{abstract}

Keywords Scoring System, Home Field Advantage Analysis, Football, Soccer

\section{Introduction}

Especially in team sports competitions and leagues, it is a known fact that home teams earn most matches regardless of the qualification of the opponent. The existence of a home field advantage is frequently investigated in contemporary sports research. The concept of home field advantage is not a new concept. When we look at the literature regarding the subject, some researches put forth that the home field advantage is found in individual sports competitions, while it is seen that team sports have more intensive research regarding home field advantage in branches such as football, handball, baseball and basketball.

Home advantage can be thought of as the percentage of the total points earned as home team and visiting team at the end of the season for the entire season [1]. Leite [2] described the concept of home advantage as consistency in winning more than $50 \%$ of matches at home. In all professional sports, especially team sports, the advantage of the home team is a known reality. Home field advantage in team sports has a significant role in determining the result of the game. Schwartz and Barsky (1977) first discovered that the home team was more successful in American football, baseball, basketball, and ice hockey [3].

The home field advantage is also an important variable influencing the result of soccer matches. The initial analysis of home field advantage in soccer was made by Desmond Morris in 1981[4]. Afterwards, some reasons related to home field advantage variable were designated by Jack Dowie in 1982, by Richard Pollard in 1986, by Stephen R. Clarke and John M. Norman in 1995, and by Richard Pollard and Gregory Pollard in 2005[5, 6, 7, 8].

In a study conducted to examine the home field advantage in European League, the home field advantage was found to be $61.2 \%$ in 380 matches in Spain, 61\% in 305 matches in Turkey, $60.7 \%$ in 240 matches in Belgium, $59.3 \%$ in 380 matches in Italy, $57.5 \%$ in 306 matches in Holland, 57\% in 380 matches in France, 56.4\% in 306 matches in Portugal, 56.2\% in 306 matches in Germany, $56 \%$ in 380 matches in England, and 55.3\% in 240 matches in Russia [2]. Based on examining 111,030 football matches played in 52 UEFA member countries between 2000 and 2010, Garcia and others [9] found the mean home advantage to be 55,6\% in their study. Seckin and Pollard [10] analyzed the last 10 seasons in Turkish 1st Football League (from 2005-2006 to 2014-2015 season) in terms of home advantage. According to this study investigating 3062 matches played in last 10 seasons between 2005-2006 and 2014-2015, the home teams won 1319 matches (43.07\%), although the home teams were defeated in 886 matches $(28.94 \%)$ and 857 matches $(27.99 \%)$ ended in a draw.

Home advantage in Turkish 1st Football League, as the second level professional football division in Turkey for the most recent 4 -year period, was $61.4 \%$. This percentage 
is almost the same as the higher level Turkish Super League. This percentage is consistent with other second level leagues in England, Germany, Spain, Italy and France [1]. Pollard [11] examined the diversity of home field advantage in soccer in different countries. He concluded that the home field advantage in the southern European countries was generally higher than in northern European countries.

In order to calculate the impact of change in scoring system on home field advantage in the English Soccer League, Pollard (1986) selected 6 seasons, 3 before the change in scoring system and 3 after the change in scoring. He found that the mean home field advantage for 3 seasons before the change in scoring $(63,3 \%)$ and for 3 seasons after the change in scoring $(63,9 \%)$ were almost the same[6]. As a comprehensive review of basic statements for the home field advantage on soccer, Pollard (2008) noted the influence of multitude, voyage factors, familiarity, referee bias, regionalism, specific strategies, mental factors, and regulation factors[12]. As one of the regulation factors affecting home field advantage, change in scoring (from 2 to 3 points for a win) in football has been analyzed by some researchers to date.

For instance, according to Dewenter [13] the 3-point system has a negative impact on the home advantage. In addition, as a result of the change in scoring, the goal difference has decreased. Dewenter (2003) argued that the decline of scored goals has caused the games to be less attractive, whereas, at the same time, the reduction in home advantage has led to exciting and less predictable games. In his research, he conducted an empirical analysis to address the question of whether the 3-point rule changed the results of the matches and significantly affected the home advantage. Under the regime of the 3-point system, the average number of goals dropped even if the average number of away goals decreased by a relative small number. He pointed out that the introduction of the 3-point system regime had some negative effects on home field advantage.

Thomas et al. [14] analyzed the home field advantage in the 1.st Division of the English Soccer League (between 1984 and 1992) and the English Football Premiership (between 1992 and 2003). They concluded that the change in scoring system in 1981 caused a remarkable decline in home field advantage.

Jacklin (2005) also analyzed home field advantage in the 1.st Division of the English Soccer League and the English Football Premiership between 1946 and 2003. He pointed out that the change in scoring in 1981 caused a decrease in the home field advantage in both soccer divisions. According to his study, the change in scoring system (changing from 2-point to 3-point) caused a 0.39 decrease in the home team wins ratio to away team wins ratio. $\mathrm{He}$ claimed that this decrease in the ratio of home team wins was very likely the result of the change in scoring system in 1981 [15].
Sanchez et al. [16] examined the impact of the change in scoring regime on home field advantage between 1980 and 2007. In the top two Spanish Soccer Divisions, change in scoring regime started in 1995-1996 season. They analyzed 20,992 games and concluded a reduction in home field advantage after the change in scoring system. By comparing the home field advantage before and after the change in scoring system, they didn't find any remarkable difference between the First Soccer Division and the Second Soccer Division. However, a significant reduction in home field advantage in both the First Soccer Division (decrease from 66,3\% to 62,1\%) and Second Soccer Division (decrease from 65,6\% to 59,5\%) was found after the change in scoring regime. In addition to this study, based on home field advantage data comparison by Thomas et al. (2004) and Pollard and Pollard (2005) on Spain and English football leagues, Sanchez et al. (2009) argued that higher home advantage rate in Spain was related to the delay in introducing the 3-point system, since Spain introduced 3-point system 14 years later (1995) than England.

Saavedra et al. [17] conducted a study (80 seasons and 22,015 games) to analyze the home field advantage of the Spanish Soccer First Division from 1928 to 2011. They found that the home field advantage is 70,8 in the highest category during the period when a victory was worth 2 points (1928-1995) and 56,7 when a victory was worth 3 points (1995-2011). Accordingly, the advantage of playing at home existed and was significant, both when a win was worth 2 points and when it was worth 3 points. However, the values of the home advantage were lower with the change in the point system, changing from 2 to 3 points for a win [17].

As can be understood from the above studies, the transition from the 2-point system to the 3-point system in football led to a decrease in the ratio of the home field advantage in all leagues in general. If the visiting team wins 3 points when they win the game in this case, it may cause them to be more willing to win. When the visiting team starts to find a position for more goals during the match, it makes the match more enjoyable for the spectators. The change in the point system in football could also have been influenced by varying tactical insights of the teams as well as by the footballers' layout on the field. The visiting teams adapting to it in the course of time, the 3-point system has ensured that they get more points in the matches they play away. As a result, the 3-point system is one of the variables that influence the reduction of the host's advantage. The Turkish Football (Soccer) League, first established in 1959 as National League, used 2-point system between 1959-1960 and 1986-1987 seasons. The 3-point system, starting first in England in 1981, has been used in Turkey since 1987-1988 season. According to the change in scoring, the winning teams earn 3 points instead of 2 points. No change exists for defeated teams, draw teams, defeated teams earn 0 
point, and draw teams earn 1 point. In order to provide an impartial calculation of home field advantage in the Turkish soccer divisions, teams play twice in a season. As a rule, teams play once in their home field and play once in the soccer field of the visiting team.

Four hypotheses were tested in this study. $\mathrm{H} 1$ : there is a significant difference between the home field advantage rates before and after the change in scoring system. $\mathrm{H} 2$ : there is a significant difference between the home team's total points at home field before and after the change in scoring system. $\mathrm{H} 3$ : there is a significant difference between the home team's total points at visiting team's field before and after the change in scoring system. H4: there is a significant difference between the home team's total draw points at home before and after the change in scoring system.

\section{Materials and Methods}

The sampling of this study consisted of 58 Turkish Soccer League seasons from 1959 to 2017. The point system in Turkey was changed in 1986-1987 season. 2-point system was used in 28 seasons out of 58 seasons, and 3-point system was used in remaining seasons. A total of 33,244 games were analyzed in this study. The website of Turkish Professional Soccer League provided retrospective data for the study.

The Turkish Professional Soccer League employed 2-point win system from the foundation year of 1959 to 1987. According to this system, the winning team got 2 points and the teams got 1 point each in the event of a tie, while the losing team got nothing. The 3-point system started in 1987-1988 season in Turkish Professional Soccer League. The winning team gets 3 points in this system, while the teams get 1 point in the event of tie. The losing team gets nothing in this system, too.

Teams play each other twice each season in the Turkish Professional soccer leagues. They play once at home field and play once away. This playing strategy contributes to the balance of the system and contributes to the neutrality of the home advantage.

Table 1 provides information regarding how many matches were played with how many teams and how many matches were analyzed at the end of the season. (www.tff.org, Turkish Football Federation website)

In order to evaluate home advantage in this study, Pollard and Pollard's (2005) method was used [8]. According to them, home field advantage is the scores earned at home field throughout a season. It is computed as a percentage of the earned scores at home field and earned scores in visiting team's field. Accordingly, Sanchez et al. [16] made an inference that if scores earned at home field and scores earned in visiting team's field equaled to $50 \%$, meaning that there is no home field advantage.
Table 1. Turkish Super League (Independent Group t Test)

\begin{tabular}{|ccc|}
\hline Year & $\begin{array}{c}\text { Number of } \\
\text { Teams }\end{array}$ & $\begin{array}{c}\text { Number of } \\
\text { matches }\end{array}$ \\
\hline $1959-1962$ & 20 & 1140 \\
\hline $1962-1963$ & 12 & 132 \\
\hline $1963-1964$ & 18 & 306 \\
\hline $1964-1966$ & 16 & 480 \\
\hline $1966-1968$ & 17 & 544 \\
\hline $1968-1981$ & 16 & 3120 \\
\hline $1981-1982$ & 17 & 272 \\
\hline $1982-1985$ & 18 & 918 \\
\hline $1985-1987$ & 19 & 684 \\
\hline $1987-1988$ & 20 & 380 \\
\hline $1988-1989$ & 19 & 342 \\
\hline $1989-1990$ & 18 & 306 \\
\hline $1990-1994$ & 16 & 960 \\
\hline $1994-2017$ & 18 & 7038 \\
\hline Total home matches & & $\mathbf{1 6 6 2 2}$ \\
\hline
\end{tabular}

Four independent groups t-tests are conducted in order to compare the differences in the home field advantage rates before and after the change in scoring system, the differences in the total points of home team at home before and after the change in scoring system, the differences in the total points of home team away before and after the change in scoring system and the differences in total points of home team from ties at home before and after the change in scoring system. SPSS Software Version 24 was used for statistical analysis of data.

\section{Findings}

The results of the statistical analysis showed that there was a significant difference in home field advantage rates after and before the change in scoring system $(\mathrm{t}=5,041$, $\mathrm{p}=0,000)$. The average home field advantage rate in the Turkish Professional Soccer League was 66,39 percent under the system of 2 points for a win. This rate fell to 61,40 percent under the 3-point system Table 2.

Table 2. Difference in Home Field Advantage rate between Scoring System (Independent Group t Test)

\begin{tabular}{|ccccccc|}
\hline Variable & $\begin{array}{c}\text { Point } \\
\text { System }\end{array}$ & $\mathrm{N}$ & Mean & $\mathrm{SD}$ & $T$ & $p$ \\
\hline$H_{A R^{*}}$ & $2 p s^{* *}$ & 28 & 66,3937 & 4,3658 & 5,041 & 0,000 \\
\hline & $3 p^{* * *}$ & 30 & 61,4074 & 3,1014 & & \\
\hline
\end{tabular}

* HAR: Home Advantage Rate

* 2ps: 2-point System

* 3ps: 3-point System

The analysis results in table 3 reveal the significant differences between 2-point and 3-point systems in terms of total points of home team at home $(\mathrm{t}=-11,83, \mathrm{p}=0,000)$. 
The total mean of 268,21 for 2 point system for the home team at home, almost doubled to a total mean of 435,20 for 3 point system.

Table 3. Difference in the Total Points of Home Team at Home between Scoring Systems (independent group t test)

\begin{tabular}{|ccccccc|}
\hline Variable & Point System & $\mathrm{N}$ & Mean & SD & $T$ & $p$ \\
\hline TPHTH $^{*}$ & $2 p s$ & 28 & 268,21 & 51,732 & $-11,83$ & 0,000 \\
\hline & $3 p s$ & 30 & 435,20 & 55,742 & & \\
\hline
\end{tabular}

* TPHTH: Total Points of Home Team at Home

In table 4 , there is a considerable increase in total points of home team away after the 1987-1988 season. There is a significant differences between 2-point system and 3-point system in terms of total points of home team earned away $(\mathrm{t}=-15,79, \mathrm{p}=0,000)$. The mean total points of home team away can be seen to be 95,07 under 2-point system. However, the average is 246,30 under 3 -point system.

Table 4. Difference in the Total Points of Home Team Away between Scoring Systems (independent group t test)

\begin{tabular}{|ccccccc|}
\hline Variable & Point System & $\mathrm{N}$ & Mean & SD & $T$ & $p$ \\
\hline TPHTA $^{*}$ & $2 p s$ & 28 & 95,07 & 40,718 & $-15,79$ & 0,000 \\
\hline & $3 p s$ & 30 & 246,30 & 31,201 & & \\
\hline
\end{tabular}

* TPHTA: Total Points of Home Team Away

In table 5 , there is also a significant differences between 2-point system and 3-point system in terms of total points of home team from ties at home $(\mathrm{t}=4,278, \mathrm{p}=0,000)$. The mean point was 89,64 under 2-point system while the mean point is 73,40 under 3-point system.

Table 5. Difference in the Total Points of Home Team from Ties at Home Between Scoring Systems (independent group t test)

\begin{tabular}{|ccccccc|}
\hline Variable & Point System & $\mathrm{N}$ & Mean & SD & $T$ & $p$ \\
\hline TPHTT $^{*}$ & $2 p s$ & 28 & 89,64 & 17,720 & 4,278 & 0,000 \\
\hline & $3 p s$ & 30 & 73,40 & 9,800 & & \\
\hline
\end{tabular}

* TPHTT: Total Points of Home Team from Ties at Home

All home field advantage values of the 58 Turkish Soccer League seasons were remarkably higher than $50 \%$ (Table 6-7). Values higher than $50 \%$ for each year showed the occurrence of home field advantage. Regarding the different periods based on scoring system in the Turkish Soccer League, the home field advantage average was $66,39 \%$ before change in scoring system (Table 6). However, the home field advantage average in the period of 3-point system was $61,40 \%$ (Table 7).
Table 6. Home Field Advantage Rate in Turkish soccer League (1959-1987)

\begin{tabular}{|c|c|c|c|c|c|}
\hline Seasons & Games & $\mathbf{H}^{*}$ & $A^{* *}$ & $T^{* * *}$ & HFA(\%) \\
\hline $1959-1960$ & 380 & 346 & 208 & 103 & 59,08 \\
\hline $1960-1961$ & 380 & 332 & 174 & 127 & 60,39 \\
\hline 1961-1962 & 380 & 350 & 162 & 124 & 62,37 \\
\hline $1962-1963$ & 132 & 128 & 52 & 42 & 64,39 \\
\hline 1963-1964 & 306 & 272 & 142 & 99 & 60,62 \\
\hline 1964-1965 & 240 & 234 & 78 & 84 & 66,25 \\
\hline 1965-1966 & 240 & 204 & 142 & 67 & 57,29 \\
\hline 1966-1967 & 272 & 242 & 102 & 100 & 62,87 \\
\hline $1967-1968$ & 272 & 266 & 106 & 86 & 64,71 \\
\hline 1968-1969 & 240 & 234 & 86 & 80 & 65,42 \\
\hline $1969-1970$ & 240 & 234 & 82 & 82 & 65,83 \\
\hline $1970-1971$ & 240 & 234 & 74 & 86 & 66,67 \\
\hline $1971-1972$ & 240 & 256 & 68 & 78 & 69,58 \\
\hline $1972-1973$ & 240 & 244 & 72 & 82 & 67,92 \\
\hline $1973-1974$ & 240 & 252 & 68 & 80 & 69,17 \\
\hline 1974-1975 & 240 & 220 & 70 & 95 & 65,63 \\
\hline $1975-1976$ & 240 & 246 & 58 & 88 & 69,58 \\
\hline 1976-1977 & 240 & 244 & 64 & 86 & 68,75 \\
\hline $1977-1978$ & 240 & 270 & 58 & 76 & 72,08 \\
\hline 1978-1979 & 240 & 280 & 50 & 75 & 75,96 \\
\hline 1979-1980 & 240 & 246 & 54 & 90 & 70 \\
\hline 1980-1981 & 240 & 294 & 56 & 65 & 75,1 \\
\hline 1981-1982 & 272 & 246 & 104 & 97 & 63,05 \\
\hline $1982-1983$ & 306 & 330 & 94 & 94 & 69,28 \\
\hline 1983-1984 & 306 & 302 & 92 & 109 & 67,16 \\
\hline $1984-1985$ & 306 & 300 & 92 & 110 & 66,99 \\
\hline $1985-1986$ & 342 & 358 & 118 & 104 & 67,54 \\
\hline $1986-1987$ & 342 & 346 & 136 & 101 & 65,35 \\
\hline Total & 7596 & 7510 & 2662 & 2510 & $\dagger 66,39$ \\
\hline
\end{tabular}

* H: Number of Home Team Win

** A: Number of Away Team Win

*** T: Number of Ties

$\uparrow$ Mean Annual Home Field Advantage

As can be seen from Table 7, only the home field advantage values of first two (1987-1988 and 1988-1989) seasons and the 1994-1995 season are slightly higher than the home field advantage average $(66,39 \%)$ of the period of 2-point system (Table 6). Remaining values of the home field advantage in Table 7 are less than $66,36 \%$. 
Table 7. Home Field Advantage Rate in Turkish soccer League (1987-2017)

\begin{tabular}{|c|c|c|c|c|c|}
\hline Seasons & Games & $\mathbf{H}^{*}$ & $A^{* * *}$ & $T^{* * * *}$ & HFA(\%) \\
\hline $1987-1988$ & 380 & 594 & 240 & 102 & 67,05 \\
\hline 1988-1989 & 342 & 570 & 234 & 74 & 67,65 \\
\hline $1989-1990$ & 306 & 474 & 240 & 68 & 63,76 \\
\hline $1990-1991$ & 240 & 339 & 171 & 70 & 62,92 \\
\hline 1991-1992 & 240 & 348 & 195 & 59 & 61,57 \\
\hline $1992-1993$ & 240 & 330 & 222 & 56 & 58,13 \\
\hline 1993-1994 & 240 & 384 & 183 & 51 & 65,02 \\
\hline 1994-1995 & 306 & 504 & 204 & 70 & 67,69 \\
\hline $1995-1996$ & 306 & 441 & 270 & 69 & 60,07 \\
\hline 1996-1997 & 306 & 477 & 243 & 76 & 63,73 \\
\hline $1997-1998$ & 306 & 453 & 228 & 79 & 63,41 \\
\hline 1998-1999 & 306 & 444 & 258 & 72 & 60,99 \\
\hline $1999-2000$ & 306 & 441 & 267 & 70 & 60,26 \\
\hline $2000-2001$ & 306 & 459 & 258 & 67 & 61,81 \\
\hline $2001-2002$ & 306 & 444 & 234 & 71 & 63,51 \\
\hline $2002-2003$ & 306 & 432 & 240 & 73 & 61,4 \\
\hline 2003-2004 & 306 & 426 & 294 & 66 & 57,75 \\
\hline 2004-2005 & 306 & 453 & 252 & 71 & 61,87 \\
\hline $2005-2006$ & 306 & 384 & 294 & 80 & 55,37 \\
\hline 2006-2007 & 306 & 432 & 219 & 89 & 62,85 \\
\hline 2007-2008 & 306 & 447 & 261 & 70 & 60,97 \\
\hline 2008-2009 & 306 & 441 & 261 & 72 & 60,64 \\
\hline 2009-2010 & 306 & 411 & 267 & 80 & 58,59 \\
\hline $2010-2011$ & 306 & 405 & 282 & 76 & 57,13 \\
\hline 2011-2012 & 306 & 429 & 261 & 76 & 61,4 \\
\hline $2012-2013$ & 306 & 417 & 246 & 85 & 60,26 \\
\hline 2013-2014 & 306 & 435 & 240 & 81 & 61,65 \\
\hline 2014-2015 & 306 & 396 & 270 & 84 & 57,55 \\
\hline 2015-2016 & 306 & 432 & 252 & 78 & 60,71 \\
\hline $2016-2017$ & 306 & 414 & 303 & 67 & 56,52 \\
\hline Total & 9026 & 13056 & 7389 & 2202 & $\dagger 61,40$ \\
\hline
\end{tabular}

* H: Number of Home Team Win

** A: Number of Away Team Win

*** T: Number of Ties

$\dagger$ Mean Annual Home Field Advantage

In line with the numbers in table 8 , there is a comparison of home field advantage values between Turkish Soccer League and First Soccer Division in Spain for the period of 27 years. From 1980 to 2007, mean annual home advantage score in top level Turkish Soccer League $(63,75)$ was the same as it was in First Division Soccer League in Spain $(63,65)$. According to Sanchez et al.'s study results [16], the mean home advantage score for the period of 2-point system was $66,27 \%$. In addition, the mean home advantage score for the period of 3-point system was $62,09 \%$. Similarly, for the same period in Turkey, the home advantage mean score average for the period of 2-point system was $67,78 \%$ and the home advantage mean score average for the period of 3-point system was $62,34 \%$ (Table 8).

Table 8. Comparison of Home Field Advantage Rates between 1980 and 2007

\begin{tabular}{|cccc|}
\hline Soccer League Origin & Point System & N of Season & Mean \\
\hline $\begin{array}{c}\text { Turkey } \\
(t=3,709, p=0,001)\end{array}$ & 2-point Sys & 7 (1980-1987) & 67,78 \\
\cline { 2 - 4 } & 3-point Sys & $20(1988-2007)$ & 62,34 \\
\hline $\begin{array}{c}\text { Spain* } \\
(t=3,09, p=0,002)\end{array}$ & 2-point Sys & $15(1980-1987)$ & 66,27 \\
\cline { 2 - 4 } & 3-point Sys & $12(1988-2007)$ & 62,09 \\
\hline
\end{tabular}

* Cited from the study of Sanchez et al. (2009)

\section{Discussion and Conclusions}

The impact of the change in the scoring system on home field advantage in the Turkish Super League, the top Turkish Professional Soccer League, between the 1959-1960 season and 2016-2017 season was examined in this study. Additionally, the home field advantage comparison between Turkish Soccer League and Spanish First Division Soccer League was conducted with analogous data from a study of [16].

The results clearly show that there is a favorable advantage for home teams over $50 \%$ in the Turkish Super League in terms of home advantage. The results obtained are parallel to the findings of similar studies. Playing a match as a home team is an important factor in all seasons influencing the outcome of the match. Based on results of this study, the first hypothesis was supported. Table 2 shows that there is a remarkable difference in home field advantage values between the periods of 2-point system and 3-point system $(\mathrm{t}=5,041, \mathrm{p}=0,000)$. According to Sanchez and others (2009), every team wants to win their home field games. Based on this argument, teams usually play for a draw in the 2-point system [16]. It was effective to give 3 points for victory, because it encourages active and attack games urging the teams to win. For this reason, a remarkable difference in the home field advantage after the change in scoring system was anticipated. This finding is consistent with the study of Pollard and Pollard (2005). They found similar results in basketball, ice hockey, and soccer [8]. On the other hand, some contradictory findings were examined by Courneya and Carron (1992) and Schlenker et al. (1995) whose study findings were constant on home field advantage in the long run[18,19].There are many previous studies available related to the subject. Most of the studies have focused on the development of conceptual models that explain why the home field advantage exists in different sports over a different period. Carron et al. (2005); Courneya \& Carron 1992 stated in their studies that the home field advantage exists in all kinds of sports. In a meta-analysis study conducted for ten different sports branches by Carron et al., 2005, the home field advantage in football as a branch was found to be 
higher. When the studies related to the subject were examined, it was emphasized that the advantage of being the home team is especially important $[20,18]$.

The second hypothesis predicted a significant difference on the total score of the home team at home after the introduction of 3-point win system. The results of analysis supported this hypothesis. There was significant differences between 2-point and 3-point systems in terms of total points of home team at home $(\mathrm{t}=-11,83, \mathrm{p}=0,000)$. The third hypothesis predicted a significant difference on the total score of the home team away after the introduction of 3-point win system. The third hypothesis was also supported. There is a remarkable increase on total points of the home team away after the 1987-1988 season. There are significant differences between 2-point system and 3-point system in terms of total points of home team away $(\mathrm{t}=-15,79, \mathrm{p}=0,000)$. The mean total points of home team away was 95,07 under 2-point system. However, the average was 246,30 under 3-point system. The fact that a significant difference arises in the 2 nd and 3 rd hypotheses in the study results is accepted as normal as 3 points are won after the match in this 3 point system. This is reflected in the league table at the end of the season. As far as results are concerned, the fourth hypothesis shows us a considerable amount of variability. The fourth hypothesis predicted a significant difference on total point of home team from ties at home before and after the introduction of 3-point win system. The fourth hypothesis was also supported. Regarding the fourth hypothesis, there is also a significant difference between 2-point system and 3-point system in terms of total points of home team from ties at home $(t=4,278, p=0,000)$. The mean point was 89,64 under 2-point system while the mean point is 73,40 under 3-point system. This finding shows that in the 3-point system, the visiting teams are out on the field to win matches, not for a draw. This situation is thought to have caused changes in the tactical understanding of the visiting teams over time. It may be that the visiting teams have increased their interest in the games after the system change, which led them to struggle more and more. Increased interest may also have contributed to the development of the football economy in industrial terms. Regarding this hypothesis: As Jacklin (2005) noted, 3-point system has reduced away teams' desire for a draw [15].

According to the numbers in table 8, from 1980 to 2007, mean annual home advantage score in Turkey $(63,75)$ was the same as it was in Spain $(63,65)$. According to Sanchez et al. [16] study results, the home advantage mean score for the period of 2-point system was $66,27 \%$. In addition, the home advantage mean score for the period of 3-point system was $62,09 \%(t=3,09, p=0,002)$. Similarly, for the same period in Turkey, the home advantage mean score average for the period of 2-point system was $67,78 \%$ and the home advantage mean score average for the period of 3 -point system was $62,34 \%$. The difference between the two period is statistically significant $(\mathrm{t}=3,709, \mathrm{p}=0,001)$.
Based on home advantage data comparison on Spain and English football leagues, Sanchez et al (2009) argued that higher home advantage rate in Spain was related to the delay in introducing the 3-point system, since Spain introduced 3-point system 14 years later (1995) than England. According to the results of this comparison between Spain and England, Sanchez et al (2009) concluded that Spanish Soccer League's high home field advantage rate was because of delay in introduction to the new scoring system. Based on this study results, contrary to Sanchez et al (2009)'s inference, it is possible to say that home advantage rate in Spain is not related to the delay in introducing the 3-point system, since Spain introduced 3-point system 8 years later (1995) than Turkey. Although Spain introduced 3-point system 8 years later (1995) than Turkey, home field advantage rate of the top Turkish Soccer League was slightly higher than that of the First Soccer Division in Spain. Other factors that were not considered in the analysis might influence home field advantage rates.

The Performed observations and potential variables should be considered before a precise conclusion. Throughout the Turkish Soccer League history, several rule changes has occurred. One of the rule changes, as a disciplinary punishment, was to play match without spectators for home teams. The women and children were only allowed to watch these specific matches from 2011-2012 to 2013-2014 seasons. Another rule change occurred in away team spectator quotas. During 1980s', about half of the seating capacity of the stadium was being reserved by home team for the away team spectators in Turkey. Later, according to the rule change, at least $5 \%$ of the seating capacity of the stadium must be reserved by home team for the spectators of visiting team. From year 1959 to 2016 , at least 10 changes in foreign player quotas would be another factor affecting home field advantage difference in Turkish Soccer League. As another potential affecting factor, limiting back pass application to the goalkeeper was introduced in 1992 in Turkish Soccer Leagues. The rule was changed in order to reduce defensive play.

From 1959 to 2017, the capacities of the football stadiums were improved in Turkey; the stadiums have particularly been renovated and improved especially in recent years in every sense. Stadium renovations and the changes in enforcement of sanctions have affected home team advantage. As another rule change, electronic ticket system started on April 19, 2014 in the 2013-2014 Turkish Professional Super League season. All-seater stadiums became mandatory because each e-ticket has to be associated with a seat number according to the regulation. The amplification of the sound that is produced by the home team spectators decreases in all-seater stadiums. The psychological pressure experienced by visiting teams is expected to decrease in this manner (Sanchez et al. 2009). So, switching to e-ticketing may also be one of the factors 
influencing the home field advantage rate.

Because the results of this study are based on archive data from the Turkish Super League, the scope of the generalization of findings is limited. The point system difference, as a factor affecting this decline in Turkish Super League, supported the results of other researchers (Sanchez, et al. 2009; Jacklin, 2005; Thomas, et al. 2004). On the other hand, there have been other changes and variables affecting home field advantage throughout the Turkish Soccer League history from 1959 to now.

In future studies, it will be useful to test the effects of other variables, especially those that have direct or indirect effect on home field advantage. The difficulty in controlling for these factors suggests the need to replicate the results of the present study in different countries and leagues in order to improve our understanding of the changes that have been taking place in home advantage in football. In addition, whether or not there is a change in team layouts and tactical insights between 2 points and 3 points system should be investigated on different leagues.

\section{REFERENCES}

[1] Pollard, R. (2006). Home advantage in soccer: variations in its magnitude and a literature review of the associated factors associated with its existence. Journal of Sport Behavior, 29, 169-189.

[2] Leite, W. (2017). Home advantage: comparison between the major European football leagues. Athens Journal of Sports, 4 , $65-74$.

[3] Schwartz, B., \& Barsky, S. F. (1977). The home advantage. Social Forces, 55, 641-661.

[4] Morris, D. (1981). 'The Soccer Tribe', Jonathon Cape Publishing, London.

[5] Dowie, J. (1982). Why Spain Should Win the World Cup. New Scientists, 94, 693-695.

[6] Pollard, R. (1986). Home advantage in soccer: A retrospective analysis. Journal of Sport Sciences, 4, 237-248.

[7] Clarke, S. R., \& Norman, J. M. (1995). Home ground advantage of individual clubs in English soccer. The Statistician, 44, 509-521.

[8] Pollard, R., \& Pollard, G. (2005). Long-term trends in home advantage in professional team sports in North America and England (1876-2003). Journal of Sports Sciences, 23, 337-350.
[9] Garcia, M. S., Aguilar, O. G., Marques, P. S., Tobio, G. T., \& Romero, J. F. (2013). Calculating home advantage in the first decade of the 21st century UEFA soccer leagues. Journal of Human Kinetics, 38: 141-150.

[10] Seckin, A., \& Pollard, R. (2008) Home advantage in Turkish professional soccer. Perceptual and Motor Skills, 107, 51-54.

[11] Pollard, R. (2006). Worldwide regional variations in home advantage in association football. Journal of Sports Sciences, 24, 231-240.

[12] Pollard, R. (2008). Home advantage in football: a current review for an unsolved puzzle. The Open Sports Sciences Journal, 1, 12-14.

[13] Dewenter, R. (2003). Raising the Scores? Empirical Evidence on the Introduction of the Three-Point Rule in Portugese Football, No 22/2003, Working Paper, Helmut Schmidt University, Hamburg. Available from: https://www.researchgate.net/publication/24047782_Raisin $\mathrm{g}$ the_Scores_Empirical_Evidence_on_the_Introduction_of the Three-Point Rule in Portugese $\overline{\text { Football }}$ [accessed Jan 18 2018].

[14] Thomas, S., Reeves, C., \& Davies, S. (2004). An analysis of home advantage in the English Football Premiership. Perceptual and Motor Skills, 99, 1212-1216.

[15] Jacklin, P. B. (2005) Temporal changes in home advantage in English football since the Second World War: What explains improved away performance? Journal of Sports Sciences, 23, 669-679.

[16] Sanchez, P.A., Garcia-Calvo, T., Leo, F.M., Pollard, R., \& Gomez, M.A. (2009). An analysis of home advantage in the top two Spanish professional football leagues. Perceptual and Motor Skills, 108(3): 789-797.

[17] Saavedra G. M., Gutiérrez, A. O., Fernández, R. J.J. \& Sa Marques, P. (2015). Measuring Home Advantage In Spanish Football (1928-2011), Revista Internacional de Medicina y Ciencias de la Actividad Física y el Deporte, 15 (57): 181-194.

[18] Courneya, K. S., \& Carron, A. V. (1992). The home advantage in sport competitions: a literature review. Journal of Sport and Exercise Psychology, 14, 13-27.

[19] Schlenker, B. R., Phillips, S. T., Boniecki, K. A., \&Schlenker, D. R. (1995) .Championship pressures: choking or triumphing in one's own territory? Journal of Personality and Social Psychology, 68, 632-643.

[20] Carron, A. V., Loughhead, T. M., \& Bray, S. R. (2005). The home advantage in sport competitions: Courneya and Carron's (1992) conceptual framework a decade later. Journal of Sports Sciences, 23(4), 395-407. 